\title{
C. Bjola, Negotiation Breakthrough Analysis: The Case of Climate Negotiations
}

\section{- Article forthcoming in International Negotiation 19/1 (2014)-}

\begin{abstract}
The article fills an important gap in the literature on climate negotiations namely, the question of breakthroughs: what exactly count as breakthroughs in climate negotiations, how to measure them empirically, and what practical implications they have for the negotiation process? To address these questions, the article draws on market trading theory and develops a framework of negotiation breakthrough analysis for defining, recognizing and measuring negotiation breakthroughs. The paper argues that breakthroughs in climate negotiations occur when outcomes breach the resistance or support levels of the parties' expectations about reaching an outcome. It concludes with a discussion of the broader contributions that technical analysis can make to the theory and practice of international negotiations.
\end{abstract}

Key words: negotiation breakthrough analysis, climate negotiation technical analysis, negotiation range, complex negotiations

Word count: 10,500 


\section{INTRODUCTION}

How to recognize breakthroughs in climate negotiations? How to measure them empirically? What practical implications they have for how diplomats prepare and revise their negotiation strategies? Against the backdrop of twenty years of intense but rather unproductive negotiations of the parties to the United Nations Convention on Climate Change (UNFCCC), these questions are increasingly hard to avoid. Are climate negotiations going to eventually lead to the adoption of a legally binding agreement, which the signatory parties to the "Durban Platform for Enhanced Action" have promised to negotiate by 2015 and to put in place by 2020 ? Or negotiations will again fail to convince the United States (U.S.), China and India to cap their greenhouse gas emissions (GHG) thus leaving the resolution of the climate issue at the discretion of voluntary commitments? The short answer is that we do not know, but this is not for lack of academic effort. In fact, there is no shortage of studies examining the steady evolution and reconfiguration of the climate negotiation process. For some, the future of climate negotiations rests with conditions involving the consolidation of carbon markets (Keohane and Raustiala 2010), successfully combating the organized climate change denial (Dunlap and McCright 2011), designing a better negotiation framework (Harstad 2010), streamlining the legal instruments (Freestone and Streck 2005), strengthening the enforcement mechanisms (Barrett 2008), or putting together a more comprehensive global deal (Stern 2010).

For others, the climate regime has entered a new phase of complexity (Biermann et al. 2010 ; Hoffmann 2011 ; Oberthür and Stokke 2011) that makes more difficult for diplomats and policy-makers to steer the negotiation process in a particular direction. This is so because of the increased diversification of actors (governments, international organizations, and private actors), proliferation of policies (legallybinding regulations, market-based mechanisms, voluntary initiatives) and fragmentation of institutions (multilateral, sub-national, regional, or private schemes) underpinning the global climate regime. In 
other words, even if the international and domestic conditions underpinning the climate agenda would somehow come together in a positive configuration, the outcome of UNFCCC negotiations would likely remain uncertain due to the growing complexity of the climate regime. What seems therefore to be lacking is a conceptual framework that would allow us to get an analytical grip of the likely evolution of climate negotiations, in general, and of the conditions for potential breakthroughs, in particular.

That being said, how exactly can this be done? Climate negotiations are generally examined in a methodologically individualist fashion by backtracking negotiation outcomes to the influence of domestic actors, constituencies and institutions (Hattori 1999 ; Kanie 2003 ; Goltz 2009 ; Harris 2009 ; Christoff and EcKersley 2011 ; Michaelowa and Michaelowa 2012). This suggests that BATNA (Fisher et al. 2011) or "two level game" approaches (Putnam 1988) could provide, in principle, a good framework for theorizing negotiation breakthroughs. However, the greater the number of parties to the multilateral framework, the less reliable these methods are for predicting negotiation outcomes and by extension, negotiation breakthroughs. For example, the number of comparisons of BATNA positions required for the identification of potential zones of agreement increases from one in the case of two negotiating parties, to three for three parties, six for four parties, ten for five parties, and so on. Furthermore, if domestic constraints are also taken into consideration then the number of comparisons of two-level winsets increases from six for two negotiating delegations, fifteen for three delegations, twenty-eight for four delegations and so on. In other words, one could effectively use BATNA and the two-level game approach for theorizing potential negotiation outcomes for maximum three parties.

This article argues that it is exactly the complexity of diplomatic negotiations that holds the analytical key to understanding the dynamic of climate negotiations. Complexity is not the problem (Crump and Zartman 2003: 1), but rather the solution to understanding how negotiations evolve and the conditions 
under which they may experience breakthrough points. To develop this argument, I venture outside the traditional literature on international negotiations and draw on a set of powerful indicators used by market analysts for assessing the direction of stock/share prices. While market trading and international negotiations are two distinct fields of activity in terms of purposes, actors and methods, there are nevertheless certain important similarities between them to encourage cross-study comparison. First, the evolution of each domain is fundamentally path-dependent to the extent that decisions involving a particular trading strategy or negotiation approach are not wholly random, but rigorously circumscribed by the course of previous actions. Second, trading and multilateral negotiation processes are characterized by a high degree of complexity informed by the collective actions of many participants the individual preferences of which are often too obscure or numerous to properly systematize theoretically. Third, non-linear conditions (i.e., breakthroughs) play a crucial role both in the formation of market trends as well as in the evolution of international negotiations to the extent that they largely define the feasibility of trading and negotiation objectives respectively.

As discussed in more detail further below, the theory of market trading enjoys important conceptual advantages in dealing with complexity relative to the negotiation literature. Therefore, much insight is to be gained from applying market analytical tools to the study of climate negotiations. Market analytical tools could be used, for instance, to unpack the influence of past negotiation outcomes, to shed light on the aggregation of individual intentions into collective actions, and in so doing, to examine the conditions for the emergence of negotiation breakthroughs. To this end, the article develops a conceptual framework of negotiation breakthrough analysis (NBA) for defining, recognizing and measuring negotiation breakthroughs. Drawing on a key concept of market analysis (trading range), I argue that negotiation breakthroughs are more likely to happen when they move outside the resistance or support level underlying parties' expectations vis-à-vis the negotiation outcome. The paper is structured in three parts. The first section introduces the concept of negotiation 
breakthrough. The second part explains the relevance of technical analysis for climate negotiations and discusses some key methodological challenges. Part three tracks empirically the evolution of breakthroughs in climate negotiations through the prism of the concept of negotiation range. The article concludes with a discussion of the broader contributions that technical analysis can make to the theory and practice of international negotiations. 


\section{THEORIZING NEGOTIATION BREAKTHROUGHS}

The question of breakthroughs remains under-researched in negotiation theory, especially in multilateral and multiparty contexts. ${ }^{1}$ This situation reflects itself in the disjointed and rather sparse literature on the topic, which can be grouped in three broad categories: process-oriented studies, turning points analysis, and complexity management approaches. Building on the classical distinction between pre-negotiation, agreement and post-negotiation stages (Spector 2003), process-oriented examinations of international negotiations argue that transitions between stages is facilitated by various drivers. Time, venue, context, and treaty-design are some of the key variables to inform such events. In prolonged international negotiations, for example, internal and external factors create strategic opportunities for highly networked actors to influence state behavior and shape negotiation outcomes (Downie 2012: 311). At the same time, the longer the negotiations and the more complex, pervasive and divisive the issues under discussion, the more likely for them to elude resolution and become intractable (Lewicki et al. 2003: 40-2). Negotiations venues matter because they influence the level of support necessary for an emerging norm to advance to the "cascade stage" (Coleman 2013: 167-170). In a similar fashion, the broader context of international negotiations facilitates positive or negative linkage dynamics between distinct negotiation fora. These linkages have, in turn, "the power to halt, suspend, support or advance the interests of a national government (or any party) engaged in negotiation" (Crump 2011: 225). Finally, successful deterring of free-riding, opt-outs and leakage determines the degree to which a treaty manage to overcome resistance against its implementation (Barret 1998).

By identifying relevant scope conditions for international negotiations to advance successfully from the pre- to the post-agreement stage, process-oriented approaches make a valuable contribution to our

\footnotetext{
${ }^{1}$ For a discussion of the distinction between multilateral and multiparty negotiations, see Crump (2003).
} 
understanding of the dynamic of the negotiation process. At the same time, the conceptual link between negotiation breakthroughs and stage succession is rather problematic, primarily because it offers a weak analytical tool for discriminating between abrupt changes in the negotiation pattern and business as usual. Breakthroughs imply a non-linear development in the negotiation agenda and not merely a procedural progression between different stages of negotiation. While the literature on negotiation "stumbling blocks" promise to provide some remedy to this problem (Sjöstedt and Penetrante 2013), it is actually the field of turning points analysis (TPA) that has already delivered tangible results. For Druckman, for instance, three elements of the negotiation process are critical for understanding the formation of turning points: precipitants, process departures, and consequences. More specifically, precipitating factors (procedures, new ideas, external events) trigger sudden departures from established patterns of negotiation, which in turn facilitate progress toward or movement away from the achievement of agreement (Druckman 2001: 527). Methodologically, TPA relies on process tracing for developing detailed chronologies of the case, identifying key departures, assessing the immediate consequences for each departure, and for backward checks of the proximal events that precipitated the departure (Crump and Druckman 2012: 17).

Although it offers a conceptually innovative and methodological rigorous framework for theorizing negotiation breakthroughs, TPA advances a suboptimal explanation to the question of how precipitants trigger abrupt changes in the negotiation pattern. More precisely, like the concept of "ripeness", turning points "are known better in retrospect through analysis than in prospect during an ongoing process" (Druckman 2001: 520). It addition, TPA is arguably most effective when applied to small-N or large$\mathrm{N}$ negotiations that can be analytical reduced to a few salient features. Complex negotiation systems involving a large number of units interacting with each other in multiple fora and in serial rounds of negotiations are less amenable to TPA due to severe methodological constraints. Acknowledging this limitation, Zartman's study on International Multilateral Negotiation: Approaches to the Management 
of Complexity (1994) placed the issue of complexity management at the heart of multiparty negotiation analysis. For Zartman (2003: 179), the study of multilateral negotiations requires an understanding of how negotiators simplify, structure, and orient complexity into a process that produces an outcome. This can be achieved, for instance, through coalition building (Hampson and Reid 2003), modular design (Watkins 2003) or multistakeholder dialogue (Susskind et al. 2003). According to this theoretical paradigm, the success or failure of multilateral negotiations is the result of the way in which parties handle complexity by reducing the number of issues, positions and tasks to manageable configurations.

While the complexity school of thought does not explicitly address the notion of negotiation breakthrough, it implicitly makes the case the latter may be driven by the parties' ability to successfully cope with complexity (e.g., the more successful the parties are in reducing the complexity of their negotiation framework, the more likely for them to achieve a breakthrough). Arguably, this is insufficient for making sense of how the concept of negotiation breakthrough could theoretically capture the complexity of international negotiations. For example, when theorizing negotiation breakthroughs, should complexity be methodologically treated as a liability to be skillfully circumvented or rather as an empirical asset to be systematically harnessed? To sum up, the existing literature on negotiation breakthroughs fails to properly account for the non-linear dimension of the concept, it methodologically privileges retrospective analysis at the expense of prospective inquiry, and it provides limited insight about how complexity could be rendered empirically transparent for studying abrupt changes in negotiation patterns. In response to these limitations, I argue that in order to achieve a theoretically insightful and empirically useful definition of negotiation breakthroughs we need to re-think our understanding of the negotiation process. More specifically, I dispute the methodologically individualist treatment of international negotiations as discrete events in which 
actors attempt to aggregate, from scratch, pre-defined individual preferences into a mutually acceptable outcome.

This is often not the case as many international negotiations are serial processes in which previous outcomes constrain and inform future outcomes. For example, climate talks have involved eighteen major rounds of negotiations thus far, the Doha trade negotiations led to eight meetings before they collapsed in 2008, while the European sovereign-debt crisis has triggered dozens of extraordinary and euro-dedicated summits of the European Council. In serial negotiations, diplomats do not start their talks from scratch every time they meet as their positions are being constrained by decisions taken in previous meetings. Each round of negotiation creates outcomes that change the context and the level of expectations of the parties concerning the following rounds. This is the key insight that allows us to properly theorize negotiation breakthroughs! By placing the negotiation process within the broader timeframe of serial meetings, this approach potentially enables more accurate recognitions of abrupt changes in the negotiation pattern, a sharper focus on prospective analysis, and a methodologically productive engagement with complexity. Therefore, I define negotiation breakthroughs as critical outcomes that substantially challenge parties' expectations about the ongoing process of serial negotiation.

The definition I propose presents three important analytical advantages. First, it offers a clear distinction between significant deviations in the negotiation pattern and business as usual. Breakthroughs occur when the levels of expectations generated by previous outcomes are breached as opposed to when negotiations progress from one stage to another, a transition which may actually be well anticipated. Second, instead of projecting abstract standards on the measurement of breakthroughs, this definition anchors the concept on intersubjectively defined expectations that parties share about the results of the negotiation process. This is important as it allows us to conduct 
prospective analysis by tracking the upward/downward movement of collective expectations about the ongoing process. Third, the serial dimension of the negotiation process captures the complexity of the process in a manner that maximizes the empirical accuracy of the conclusions: the larger the number of negotiation meetings, the more accurate the identification of the breakthroughs. The remaining challenge is, of course, the operationalization of the concept. The next section explains the relevance of technical analysis for climate negotiations and discusses some of the methodological challenges for conducting empirical research of negotiation breakthroughs. 


\section{APPLYING TECHNICAL ANALYSIS TO CLIMATE NEGOTIATIONS}

\section{1. What is Technical Analysis?}

Identifying and forecasting negotiation breakthroughs are not easy undertakings. Many would argue, even impossible. However, the entire business of market trading is based on this premise: trends in the collective negotiation of stock prices by market players can be properly identified and the results (i.e., potential breakthroughs) can then be used to inform decisions about when to sell and buy financial assets and commodities. Surely, the history of market trading is full of cases of lost fortunes on account of inaccurate readings of market trends (the financial crisis in 2008 being the most recent example), but most often than not the tools used for market analysis have proved reasonably reliable. ${ }^{2}$ The objective of this article is not, of course, to provide an in-depth overview of the various trading tools, strategies and instruments that are being used by investment banks, private equity firms and hedge funders around the world to maximize their capital gains. The goal is rather to identify key concepts in market analysis, which have the strongest potential to shed light on the likely evolution of international negotiations in general and of climate talks in particular.

Broadly speaking, market trading theory is divided between two schools of thought: fundamental and technical analysis. The first one concerns itself with the use of "hard" economic data such as net profit margin, price/earnings ratio, return on average assets, debt to equity ratio and so on, with the purpose of screening assets that have the strongest likely return value in medium and long term. Technical analysis, on the other hand, focuses on price behavior using various indicators for identifying patterns, moving averages, oscillators, support and resistance levels that can signal good entry and exit points

\footnotetext{
${ }^{2}$ The growing revenue contribution of the financial sector of the City of London to the UK economy provides a powerful confirmation of the empirical validity of market analysis. Market trading and related financial services in the city of London accounted for $11.2 \%$ of total UK government tax receipts in 2010 despite the negative impact of the financial crisis in 2008-9 (City of London 2010: 3).
} 
for trades in the short-term. In practice, however, successful traders use a combination of both models. Fundamental analysis serves to determine the market direction in which to trade, while technical analysis helps identify the time for the entry and exit of such trades (Schwager 1999: 3). The parallel with climate negotiations is relatively transparent. A fundamental analysis of climate talks would be about assessing the likely negotiation position of a country based on its particular matrix of economic, environmental, political or strategic interests. This approach informs the vast majority of studies of international negotiations. Outcomes are explained by reference to various combinations of individual preferences in line with well-established negotiation models, such as the two-level game (Putnam 1988), the best alternative to a negotiated agreement - BATNA (Fisher et al. 2011) or various models of multiparty negotiations (Crump and Glendon 2003).

The "fundamental" approach has obvious merits for understanding the distribution of the bargaining power among the UNFCCC parties and the checks this distribution places on the process and outcome of climate negotiations. By understanding the "fundamentals" (economic costs, political constraints, institutional limitations) of the negotiation positions of the key players, relevant points of converge/divergence between parties can be identified. The resulting "zone of agreement" may then shed light on what needs to be done to put the climate talks on a positive track. Nevertheless, by focusing on individual positions, this approach fails to properly take into account the aggregate influence of past rounds of negotiations and the impact they may have on future climate talks. In so doing, they fail to account for how negotiation breakthroughs take place. The Kyoto Protocol, the Bali Roadmap, the Cancun Agreements, the Copenhagen Accord, the Durban Platform and the other outcomes reached by the UNFCCC Conferences of the Parties (COP) - each has added a new layer of objectives, commitments and institutions that constrain the actions of the parties, frame their positions in the next stages of negotiations, and define the expectations for potential breakthroughs. Put differently, the study of climate talks needs to take into account the broader context each round of 
negotiations helps create for how parties define and pursue their individual interests in the following rounds.

This is why a technical analysis could prove extremely useful for the study of climate negotiations. Simply put, technical analysis is about examining the overall pattern of the past negotiation outcomes and using the results for identifying conditions for potential breakthroughs. The difference between the "fundamental" and "technical" approach for the study of climate negotiations is substantial and bears important theoretical implications. The technical approach is not ontologically informed by the preferences of individual actors, but by the history of their diplomatic interactions. In other words, negotiations are not regarded as discrete moments in time in which actors attempt to aggregate, from scratch, pre-defined individual preferences into a mutually acceptable outcome. Negotiations are instead seen as a process in which outcomes of previous meetings largely constrain and inform individual preferences and the negotiation parameters of the next meetings. This method has been largely neglected by negotiation scholars, an omission this article finds to be unjustified given the significant theoretical contributions that technical analysis can make to the study of international negotiations. Before discussing these contributions in greater detail, we need first to understand the broader methodological challenges that negotiation research needs to overcome.

\subsection{Methodological challenges}

Thus far I have drawn attention to the fact that the treatment of international negotiations in methodological individualist terms hinders our effort to theorize negotiation breakthroughs and that a more systemic approach, inspired by technical analysis, could be more fruitful. This is an important double claim that needs to be properly defended. With respect to the first part of the claim, I refer to 
methodological individualism as the school of thought according to which "social phenomena must be explained in terms of individuals, their physical and psychic states, actions, interactions, social situation and physical environment" (Udehn 2001: 354). While the supporters of this view may share stronger or weaker interpretations of the degree to which "social situations" are reducible to individual preferences (Steel 2006: 442; Hodgson 2007), my main concern with this method of social explanation, especially within the context of international negotiations, is its micro-level explanatory focus. More specifically, it is generally assumed that the process of international negotiations follows a linear chain of micro-interactions (diplomats making offers, cutting deals, establishing issue-linkages etc.), and that the process itself is irrelevant or has little impact on the outcome.

By contrast, I argue that a negotiation process constitutes a macro-structure in its own right, which becomes more complex, stable and by implication, more constraining on actors' actions in a directly proportionally manner with the scope and duration of the negotiation process. In other words, while micro-level explanations can be illuminating for understanding the negotiation dynamic between small-N parties and over short periods of time, they possess lighter analytical currency for clarifying the results of large-N and prolonged negotiations. To do that, we need a method that would allow us to trace the path-dependent impact of previous rounds of negotiations on the current agenda. That being said, it is also important to be very precise about the argument I advance here. My point is not that we need to replace methodological individualism with methodological structuralism and to explain negotiation outcomes and breakthroughs solely by reference to the macro-level properties of the negotiation process. Similar to Garfinkel, I essentially argue that an "explanation seeks its own level" and "the level on which it occurs will be whatever one has the [...] structural factors that make nontrivial explanation possible" (Garfinkel 1981: 59). Put differently, the research question should drive the methodological approach and not the other way around. 
The methodological position I am therefore defending is best captured by the notion of explanatory pluralism, which asserts "that a macro- and a micro-explanation of the same social phenomenon have different but complementary virtues; which explanation is preferred in a given context depends on what first motivates the explanatory enquiry" (Marchionni 2008: 317). Given the nature of the question addressed by this study (how to recognize breakthroughs in climate negotiations?) the appropriate level of explanation cannot be one focused on the individual preferences of a few actors, but rather one that takes fully into account the complex and path-dependent structure of the climate negotiation process in creating or denying opportunities for breakthroughs. This is what makes technical analysis so relevant for the current discussion. It provides us important analytical tools by which to empirically assess the relationship between previous rounds of negotiation and breakthroughs. The concept of the negotiation range to be introduced further below captures, for instance, the spectrum of collective expectations regarding the possible outcomes of diplomatic talks. In so doing, it offers us an excellent analytical entry point for understanding the dynamic of negotiation breakthroughs.

In order to preserve the clarity and coherence of the argument, a detailed description of the methodological apparatus to be used in relation with the concept of negotiation range will be presented separately in the next section. In the remaining part of this segment, I will limit myself to making two general methodological comments. First, the examination of breakthroughs in the context of climate negotiations follows the enhanced case study approach (ECS), whereby the case material serves to illuminate the particular theoretical perspective offered by technical analysis. As Druckman points out, while the use of a few concepts limits the interpretive value of the exercise, ECS nevertheless helps demonstrate "the applicability or robustness of the theoretical concepts" (Druckman 2005: 170), which is the main purpose of this study. To this end, the paper observes the four steps that are usually deemed necessary for conducting ECS: a) selection of the case to analyze (climate negotiations); b) development of a chronology of the case (see (UNFCCC 2013)); c) description of the relevant concepts 
for the analysis (e.g., negotiation range); and, d) discussion of the conclusions that provide new insights into the case (Druckman 2005: 173)

Second, it is also important to explain the type of data points the study applies to probe the empirical validity of the concept of negotiation range for making sense of breakthroughs in climate talks. To this end, the study relies on both quantitative and qualitative data. The quantitative data set includes the number of decisions and resolutions adopted by each Conference of the Party (COP) between 1995 and 2012, the chronology and summary of which is publicly available on the UNFCCC website (UNFCCC 2013). The transparency of the quantitative data set is particularly important as it boost confidence in the final results by ensuring the reliability of the data-collection process and the replicability of the reasoning logic (King et al. 1994: 25-6). As regards the qualitative data input, it includes brief questionnaire responses of ten climate scholars and practitioners (see details in footnotes 7-8). The results of the survey are reasonably reproducible provided the selection of the subjects follows a similar purposive sampling method (see details below). The theoretical requirements guiding the data selection and aggregation process will be discussed in the next section together with the methods used in relation to the concept of negotiation range for theorizing climate breakthroughs. 


\section{ASSESSING BREAKTHROUGHS IN CLIMATE NEGOTIATIONS}

\subsection{Conceptual development}

The concept of trading range is commonly used in market analysis for tracing the evolution of stock prices over a particular period of time. In plain terms, it refers to the horizontal corridor within which stock prices fluctuate for shorter or longer periods (Schwager 1999: 51). As Fig 1 illustrates, the price of General Electric shares oscillated between \$19.5 and \$23 per unit between July 2012 and January 2013, but most of the time they stayed within the \$20-22 trading range (General Electric 2013). The upper boundary of the range (\$22) represents the resistance level, while the lower boundary $(\$ 20)$ is the support level. Support and resistance should be seen as two parts of one whole. The support level can be compared to a price floor, while resistance can be considered a price ceiling. Support can therefore be defined as the recurring upward price "pressure" exerted by expected net market buying at relatively lower prices. By the same token, resistance represents the recurring downward price "pressure" exerted by expected net market selling at relatively higher prices (Chen 2010: 58). The longer the trading range the stronger the resistance and support levels and by extension, the more significant the potential breakout.

Fig 1: Trading Range - General Electric Company (NYSE:GE) ${ }^{3}$

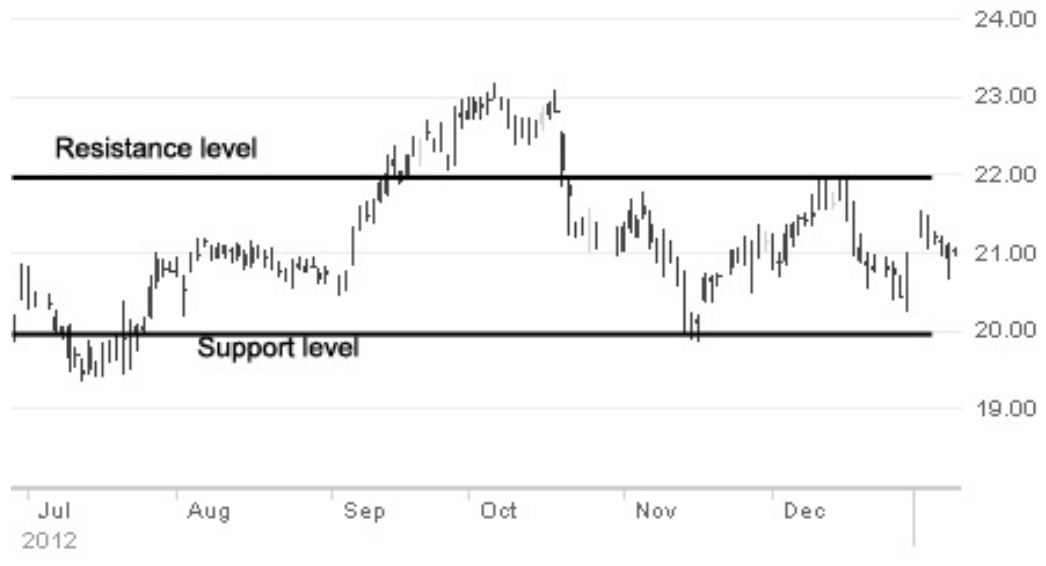

\footnotetext{
${ }^{3}$ The chart has be drawn using the online tools offered by TD Waterhouse (2013).
} 
The relevance of the concept of trading range for the study of climate negotiations stems from its capacity to render a very complex dynamic into a researchable proposition. More specifically, it theoretically anchors the concept of negotiation breakthrough to the collective expectations of the parties regarding the outcome of climate negotiations. The theoretical justification for this insight is path-dependency. The evolution of collective expectations during climate negotiations from $t$ to $t+1$ is not random, but it is shaped by the outcomes of prior rounds of negotiations. Each stage of negotiation results into new outcomes, which in turn inform parties' expectations regarding the next stages. The way in which parties then meet these expectations, or fail to do so, define the overall levels of support and resistance of the negotiations. International negotiations always have to clear disagreements, but certain obstacles are much more difficult vs. easy to overcome than others. This is why the concepts of resistance and support levels are essential for theorizing negotiation breakthroughs as they define the critical areas that parties expect to be most challenging vs. auspicious for reaching agreement.

Equally important, these levels can be ascertained even without knowing the specific individual preferences or negotiation positions of the parties. As long as the interim negotiation outcomes stay within the established levels of support and resistance, there is no breakthrough, just business as usual. However, once the levels of expectations generated by previous outcomes are breached then negotiation breakthroughs are likely to occur. Positive breakthroughs take place when negotiations move outside the resistance level as they help push the negotiation agenda forward. Negative breakthroughs (or breakdowns) take place when the support level is pierced as they prompt parties to re-visit files already agreed upon. That being said, how exactly can we measure the levels of support and resistance of climate negotiations? While market analysts rely on share prices to identify the boundaries of the trading range, diplomatic scholars require a different kind of metric to measure the evolution of international negotiations within their resistance and support levels. 
One solution is to use the documented output of negotiations (e.g., number of decisions and resolutions adopted by parties at the end of each negotiation meeting) as a measure of quantifying the gains and losses made in successive negotiation rounds. Outputs increases from meeting $M$ to meeting $M+1$ may signal the expansion of the negotiation agenda and with that the rising expectations of the parties concerning the following stage of negotiations. By contrast, diminishing outputs between $M$ and $M+1$ may indicate the parties' exhaustion or declining satisfaction with the negotiation process. However, quantitative metrics are insufficient for making sense of how well negotiations proceed as the substance of the adopted decisions and resolutions is as important as their number. Do parties properly address the issues raised in previous negotiations or they simply overlook them for lack of consensus? Do new decisions help consolidate prior outcomes or they open files that make more difficult for the negotiating parties to put in practice decisions already adopted? In short, do output increases strengthen, weaken or make no qualitative difference for how the negotiation process evolves from one meeting to another?

\subsection{Methods}

The methodology required to address these questions from the perspective of climate negotiations involves two important components: data-collection and index-formation. The documented output of climate negotiations is the easiest to collect as the UNFCCC has made available online the chronology and summary of all COP meetings and outcomes (UNFCCC 2013). For reasons of data consistency, the empirical analysis includes only COP outputs since unlike COP decisions that go back to 1995 , CMP decisions are available only since 2005, the year when the Kyoto Protocol entered into force. ${ }^{4}$

\footnotetext{
${ }^{4}$ If the analysis includes both COP and CMP decisions, COP decisions prior to 2005 have to be dropped out. This move would reduce the number of negotiation rounds in the data set from eighteen to eight, a fact that would significantly narrow the range of relevant observations and by extension the reliability of the final results.
} 
As specified in the UNFCCC summary, COP 1 concluded with 22 items ( 21 decisions, 1 resolution), COP 2 with 18 items ( 17 decisions, 1 resolution) and so on. ${ }^{5}$ Once so collected, the data is then rescaled on a numerical scale between -2 and $2^{6}$ so that the values of the variable measuring the quantitative aspect (Output) of the evolution of climate negotiations can be compared with the values of the variable measuring its qualitative dimension (see Q-Factor below) on the same scale. The first column (Output) in Table 1 captures the quantitative component of the negotiations by including the total number of decisions and resolutions adopted by each COP. The second column (N-Output) includes the rescaled values of the Output variable on the new scale between -2 and +2 .

The qualitative dimension of climate outcomes is captured by the Q-Factor (third column in Table 1) through a five-point summated rating scale (SRS), also known as Likert scale. Given its good reliability and validity in capturing individual attitudes (Spector 1992: 2), SRS is being applied here for measuring the qualitative difference between the output of $\mathrm{COP}_{n+1}$ vs. $\mathrm{COP}_{\mathrm{n}}$. The construction of the scale is based on the following rule: if $\mathrm{COP}_{\mathrm{n}+1}$ concludes with a more robust outcome than $\mathrm{COP}_{\mathrm{n}}$, it receives a score of 1 or 2 depending on the perceived strength of the outcome. If it makes no qualitative difference, it receives a score of zero. If the $\mathrm{COP}_{n+1}$ outcome weakens the $\mathrm{COP}_{\mathrm{n}}$ decision, it then receives a score of -1 or -2 proportionally with the perceived intensity of the loss. While the survey instructions were formulated in clear, transparent and concise terms ${ }^{7}$ in line with customary criteria of

\footnotetext{
${ }^{5}$ The UNFCCC summary lists only 7 items under COP6 (4 decisions, 3 resolutions) (UNFCCC 2013), but those refer only to the COP6a meeting held in the Hague in Nov 2000. Table 1 combines the 7 item output of COP6a with the two decisions adopted at COP6b held in Bonn in July 2001 (UNFCCC 2001).

${ }^{6}$ Rescaling involves setting the smallest observed value equal to the minimum value of the new scale, the largest observed value equal to the maximum value in the new scale, and fitting all other values in between while maintaining the relative distances between them (Hartwig and Dearing 1979: 60-1). The rescaling formula applied for calculating the N-Output variable is the following: Rescaled Value $=a+(x-A)(b-a) /(B-A)$, where $x=$ Output value to be rescaled, $a=-2$ (min value of the new scale), $b=2$ (max value of the new scale), $A=9$ (min Output value), $B=41$ (max Output value). For example, the rescaled value of the Output variable for COP1 is $-2+(22-9)[2-(-2)] /(41-9)=-0.375$.

${ }^{7}$ The interviewees were given the following instructions: "One a scale from -2 to +2 , please give your assessment of the outcome of each COP in comparison to the previous COP (see attached the summary of COP outcomes). For example, if you think that COP 7 moved the climate agenda forward comparing with the outcome of COP 6 , please enter +1 (progress) or +2 (significant progress) depending on how beneficial you perceive the COP 7 outcome to have been for climate negotiations relative to that of COP6. If you think it made no significant difference please enter 0. If you think it COP7 weakened the outcome of COP6, please enter -1 (minor setback) or -2 (major setback)."
} 
construct validity (Alasuutari et al. 2008: 315 ), the SRS values (-2 for major setback, -1 for setback, 0 for no change, 1 for progress, 2 for significant progress) were left intentionally unspecified. The reasoning behind this was to give respondents minimal discretion in assessing the qualitative difference between $\mathrm{COP}_{\mathrm{n}+1}$ vs. $\mathrm{COP}_{\mathrm{n}}$ outputs as opposed to indirectly encouraging them to reproduce the author's evaluation preferences.

Table 1: Quantitative and qualitative outputs of UNFCCC COPs (1995-2012)

\begin{tabular}{|c|c|c|c|c|c|c|c|}
\hline $\begin{array}{c}\text { Negotiation } \\
\text { round }\end{array}$ & Year & Location & $\begin{array}{c}\text { Output } \\
\text { (I) }\end{array}$ & $\begin{array}{c}\mathbf{N}- \\
\text { Output } \\
\text { (II) }\end{array}$ & $\begin{array}{c}\text { Q- } \\
\text { Factor } \\
\text { (III) }\end{array}$ & $\begin{array}{c}\text { Q- } \\
\text { StDev } \\
\text { (IV) }\end{array}$ & $\begin{array}{c}\text { Progress } \\
\text { Factor(PF) } \\
\text { (V) }\end{array}$ \\
\hline COP1 & 1995 & Berlin & 22 & -0.375 & 1 & 0.71 & 0.31 \\
COP2 & 1996 & Geneva & 18 & -0.875 & 0.25 & 0.89 & -0.31 \\
COP3 & 1997 & Kyoto & 19 & -0.750 & 2 & 0.00 & 0.63 \\
COP4 & 1998 & Buenos Aires & 21 & -0.500 & 0.11 & 0.60 & -0.19 \\
COP5 & 1999 & Bonn & 22 & -0.375 & 0 & 0.71 & -0.19 \\
COP6a+b & 2000 & The Hague, & 9 & -2.000 & 0.5 & 0.71 & -0.75 \\
& 2001 & Bonn & & & & & \\
COP7 & 2001 & Marrakech & 41 & 2.000 & 0.89 & 0.93 & 1.44 \\
COP8 & 2002 & New Delhi & 26 & 0.125 & -0.22 & 0.44 & -0.05 \\
COP9 & 2003 & Milan & 23 & -0.250 & 0.67 & 0.50 & 0.21 \\
COP10 & 2004 & Buenos Aires & 19 & -0.750 & 0.22 & 0.67 & -0.26 \\
COP11 & 2005 & Montreal & 16 & -1.125 & 1.56 & 0.53 & 0.22 \\
COP12 & 2006 & Nairobi & 10 & -1.875 & 0.89 & 0.60 & -0.49 \\
COP13 & 2007 & Bali & 15 & -1.250 & 1.56 & 0.53 & 0.15 \\
COP14 & 2008 & Poznan & 10 & -1.875 & 0.70 & 0.67 & -0.59 \\
COP15 & 2009 & Copenhagen & 14 & -1.375 & -0.30 & 1.42 & -0.84 \\
COP16 & 2010 & Cancun & 13 & -1.500 & 0.50 & 0.97 & -0.50 \\
COP17 & 2011 & Durban & 20 & -0.625 & 0.50 & 0.85 & -0.06 \\
COP18 & 2012 & Doha & 27 & 0.250 & 0 & 1.25 & 0.13 \\
\hline
\end{tabular}

The Q-Factor values represent the average responses of ten climate scholars and practitioners, which were selected through a non-probability, purposive sampling method. ${ }^{8}$ The choice of the method was

\footnotetext{
${ }^{8}$ Out of the ten experts interviewed for this study, three are senior academics who have published and taught climate negotiation \& politics, three are senior practitioners, two are Ph.D. students with strong climate expertise, and two are environmental consultants working for the UN Environment Programme and the UN Research Institute for Social Development respectively. Among the senior practitioners, one serves as a Senior Adviser on Climate Negotiations for the European Commission, the second has led the Institutions and Governance Program of the World Research Institute, and the third works as Senior Climate Policy Expert for the Ministry of National Development of a UNFCCC party. The inclusion of the Ph.D. students and environmental consultants alongside senior academics and practitioners in the survey
} 
informed by the need to ensure specialist knowledge of the research issue and the capacity of the responders to participate in the research (Jupp 2006: 244). The cross-section of climate experts also facilitates a balanced view of climate negotiations from both theoretical and practical perspectives. Each responder was presented with the UNFCCC summary of COP outcomes (see (UNFCCC 2013)) and asked to qualitatively compare the outcome of each COP relative to the previous one on the SRS scale described above (see footnote 7). The summary was primarily intended to serve not as a closed assessment grid but as a useful reminder of the evolution of climate talks to be used by the interviewees as general guidance in their assessment of the subject. The standard deviation of the responders' rankings builds confidence in the validity of the results (Q-StDev, fourth column in Table 1). With two exceptions (COP 15 and Cop 18), the responders have produced similar qualitative assessments of COP outcomes. The survey responses thus approximate a normal distribution of the data and hence the Q-Factor average is a good estimate of the responders' views (Vogt 2005: 307).

The final methodological step is the creation of an index combining the quantitative and qualitative measures of climate negotiation outcomes. The matter requires some preliminary clarifications as the quantitative indicator (Output) is the product of a numerical scale, while the qualitative variable (QFactor) is the result of an ordinal scale. As a first step to ensure data compatibility with the Q-Factor, the Output values have been rescaled into the $-2 / 2$ scale (N-Output). This operation does not affect the integrity of the N-Output data set. A simple test using the Rank function in Excel confirms, for instance, that the rank of the values in the data set has remained identical before and after rescaling. While being commonly used as a social research method, the conversion of ordinal values into their numerical equivalent is, however, more problematic. The process of assigning numerical (nominal or ordinal) values to data conceived as not numerical (e.g., ordinal) is known as quantitizing and it is done for the purpose of putting qualitative data into a form amenable to statistical assimilation 
(Sandelowski et al. 2009: 209-210). The problem arises from two measurement concerns: first, that the conceptual distances between scale items might not be equal and second, that the respondents might not view and interpret the scale in a similar fashion (Granberg-Rademacker 2010: 75).

While the academic debate vigorously continues about how best to address the limitations of quantitizing (Jamieson 2004 ; Carifio and Perla 2007), it nevertheless offers us sufficient theoretical insight for justifying the merging of N-Output and Q-Factor variables. First, it is important to note the qualitative indicator (Q-Factor) does not only capture a manifest variable (the qualitative difference between $\mathrm{COP}_{n+1}$ vs. $\mathrm{COP}_{n}$ ), but it also provides indirect information about a latent variable (the evolution of parties' expectations regarding the outcome of the negotiations). As the latent variable is typically considered to be interval scaled and hence continuous (Harwell and Gatti 2001: 110), a partial defence to the first measurement objection may thus follow from this insight. Second, the small size of the sample and the adequate standard deviation scores of the responders' rankings (Q-StDev) offer a satisfactory level of assurance against the second objection regarding scale interpretation. For larger sample sizes, due to the greater risk of statistical error, it might be useful, though, to engage in more sophisticated tests of ordinal data reliability and scale conversion (Granberg-Rademacker 2010 ; Gadermann et al. 2012).

With these considerations in mind, I then proceed to creating the Progress Factor index (PF) by averaging the Q-Factor and N-Ouput values (see fifth column in Table 1). The development of the PF index rests on the implicit assumption the quantitative and qualitative variables bear equal weight on the measurement of negotiation outcomes. In other words, I find no reason to attach different weights to the two variables (Q-Factor and N-Ouput) since the documented output of negotiations and the qualitative scores of climate experts take distinct but equally important measurements of the parties' expectations concerning the outcome of climate talks. Having described the methodological apparatus, 
the article focuses in the next section on examining how the PF index can be applied to identifying breakthroughs in climate negotiations.

\subsection{Negotiation breakthrough analysis}

A close reading of the data in Table 1 confirms the empirical validity of the PF index for tracing and assessing the evolution of climate negotiations. For example, the PF index shows that despite their decisional output, $\operatorname{COP}$ 4, 5, 8 and 17 have made little qualitative difference for the overall negotiation process. This finding is in line with the empirical evidence. The Buenos Aires Plan of Action adopted by COP4 merely established deadlines for finalizing the outstanding details of the Kyoto Protocol. COP 5 produced guidelines for the preparation of national communications of GHG emissions by developed countries. COP8 concluded with a simple Declaration on Climate Change and Sustainable Development, while COP 17 ended with a vague commitment of the parties about adopting a universal climate agreement by 2015. By contrast, the PF index suggests COP 3 and especially COP 7 have significantly advanced the climate agenda. Again, the empirical evidence supports this conclusion. COP3 produced binding GHG reduction obligations for developed countries, while COP7 established instruments of implementation of these commitments (UNFCCC 2013).

The PF index also finds COP 6a and COP 15 to have led to setbacks for the negotiations. As the empirical evidence indicates COP 6a was suspended because parties could not reach an agreement about how developed countries should fulfill their commitments. Despite agreeing to mobilize climate adaptation finance for developing countries up to US\$100 billion a year by 2020 (UNFCCC 2009: 7), COP15 nevertheless failed to achieve a binding agreement for long-term action. The negotiating parties instead decided to substitute legally binding reduction targets with voluntary pledges, both for 
developed and developing countries. Finally, the negotiations' upward move after Copenhagen indicated by the PF index can be explained by the extension, at COP 18, of the Kyoto Protocol to a second commitment period with a view to reduce the overall amount of GHG emissions by at least 18 per cent below 1990 levels between 2013 to 2020 (UNFCCC 2012). In short, the PF index proves itself a valuable and reliable instrument for empirically measuring progress in climate talks.

The fact that the PF index enjoys strong empirical validity in assessing the contribution of each COP to the overall progress of climate negotiations is clearly important, but this tells us little about how to recognize breakthroughs. In other words, we still need to develop a method by which to apply the PF index so that we can clearly identify potential breakthrough points during climate negotiations. As discussed above, breakthrough analysis requires a clear understanding of how the levels of collective expectations generated by previous negotiation outcomes are being breached in succeeding meetings in a manner that either push the negotiation agenda forward (positive breakthroughs) or backwards (negative breakthroughs). By reliably determining the resistance and support levels of the climate negotiation range, the PF index helps locate such breaches in the levels of collective expectations. This can be accomplished in two steps (see Fig. 2). First, by plotting PF values on a two dimensional graph, whereby the vertical axis marks increments of the $-2 /+2$ scale, ${ }^{9}$ while the horizontal axis reflects the temporal dimension of climate negotiations. Second, we draw the upper and lower boundaries of the negotiation range either by using the trendline options available in any charting software package ${ }^{10}$ or by manually connecting as many points of close high and low PF value respectively.

\footnotetext{
${ }^{9}$ The absolute values of the scale $(-2 .-1.0,+1,+2)$ have no particular meaning. What matters is the position of PF values relative to each other since this reflects the upward or downward trend of climate outcomes.

${ }^{10}$ In this particular case, the upper boundary is best approximated by an exponential function $(y=0.6891 \mathrm{e}-0.092 \mathrm{x}$; $\left.\mathrm{R}^{2}=0.3889\right)$, while the lower boundary is defined by a polynomial function of second degree $(\mathrm{y}=0.0017 \mathrm{x} 2-0.0473 \mathrm{x}-$ $0.1304 ; \mathrm{R}^{2}=0.0846$ ). Both functions have been calculated using Microsoft Excel.
} 
Fig. 2: Climate Negotiation Range - Resistance and Support Levels

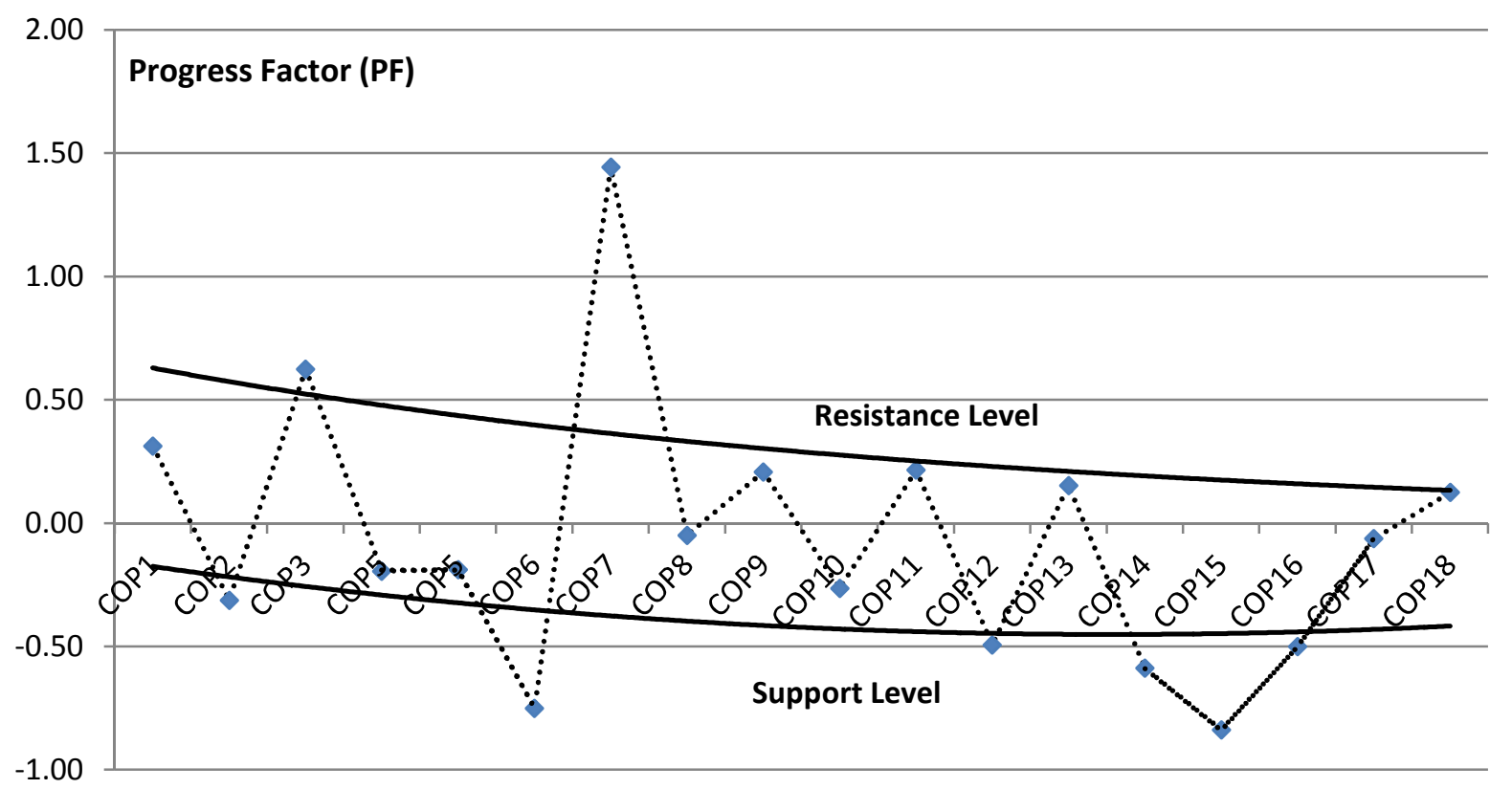

The application of technical analysis to climate negotiations thus yields a remarkable insight. It is not only that financial securities trade in ranges, but international negotiations can be conceptualized in a similar fashion. The key to understanding this point is the high degree of complexity that characterizes serial and multilateral negotiations. The longer the negotiations and broader their membership, the larger the number of observations (negotiation outcomes) and the more constraining the negotiation process on actors' positions. It is exactly this configuration of complexity-inducing factors that enables us to accurately recognize significant changes in the negotiation pattern that is, breakthroughs. Equally importantly, the concept of negotiation range helps us make sense of the evolution of climate negotiations in a highly parsimonious manner. While it is theoretically conceivable to achieve the same results using classical negotiation theory (BATNA, TPA or the two-level game model), the dual concept of support and resistance levels allows us to identify the most likely negative and positive breakthroughs without any need for engaging in complicated examinations and assessments of the negotiation preferences of large-N parties. 
More concretely, negotiation breakthrough analysis (NBA) makes a three-fold contribution to our understanding of the evolution of climate talks. First and most importantly, by uncovering the pivotal moments when the resistance and support level of collective expectations have been significantly breached, NBA clearly and reliably identifies the points of positive or negative breakthrough during the negotiations. As shown in Fig 2, COP 6(a) and COP 15 produced outcomes well outside the support level, while COP3 and especially COP7 broke the resistance level. From a practical perspective, these observations have important implications. By knowing that a particular negotiation outcome has moved outside the support or the resistance level, a diplomat is in a stronger position to realize the seriousness of the situation, especially when that has occurred in a relatively advanced stage of the serial negotiation process. The breakthrough simply means the outcome cannot be considered business as usual, but rather a significant departure from the negotiation pattern. This requires, in turn, a corresponding adjustment of the negotiation strategy. For example, in the case of a negative breakthrough, it would probably make better sense for the diplomat to recommend her government to follow rather than lead other parties in enforcing the convened recommendations. By contrast, in the case of a positive breakthrough, the diplomat should take note of the growing consensus and move swiftly to coordinate actions that can facilitate the implementation of the agreement.

Second, NBA can be applied for narrowing down the issues that have proved the least vs. most problematic for the negotiators to agree upon. For example, the negotiation outcomes of COP 2, 12, and 16 indicate a good level of support among developed countries for providing financial and technological assistance to developing countries for climate adaptation purposes. By contrast, the negotiation outcomes of COP 11, 13 and 18 suggest a good level of interest among UNFCCC parties in long-term cooperative action for reducing GHG global emissions, but steady resistance against extending the asymmetrical distribution of emission reduction commitments between the developed and developing countries beyond 2012. Again, from a practical perspective, a diplomat can use the 
support and resistance levels for identifying the issues that are most/less likely to advance the negotiations. This way, the diplomat can make better use of critical resources (time, expertise, personnel) in the pursuit of her negotiation objectives. For example, she can focus her attention on issues that can be delivered, whether they deal with technological assistance, climate adaptation and so on, while gradually building the necessary network of support for negotiating the "hard" issues (e.g., binding GFG reduction commitments) under more favorable circumstances.

Third, the methodology described in this paper for constructing negotiation ranges and identifying breakthroughs is relatively simple and hence, it can be successfully applied by anyone, scholars and practitioners alike, regardless of their level of expertise. This creates a clear competitive advantage for the NBA method over alternative models. As mentioned above, BATNA and the two-level game approach could be used for theorizing negotiation outcomes for maximum three parties as they face serious methodological constraints when applied to serial and complex international negotiations. Turning point analysis is more successful in addressing multilateral negotiations (Crump and Druckman 2012), but it requires elaborate coding judgments, cross-tabulations and process-tracing procedures (Druckman 2001: 528-530). By contrast, NBA requires the analyst to be familiar with some basic procedures of data collection, statistical interpretation and graph design. In other words, once the data-collection step is completed, NBA can deliver solid results straightaway without any need for engaging in sophisticated statistical methods of data aggregation, comparison and interpretation.

On the critical side, NBA faces several empirical and conceptual limitations. For example, breakthrough analysis produces empirically relevant results only if the negotiations have a sufficiently long track record (e.g., at least three successive rounds of negotiation). The larger the number of negotiation outcomes, the more accurate the support and resistance levels and by extension, the more precise the identification of negotiation breakthroughs. Multilateral negotiations with short track 
records tend to create weak constraints upon the parties, giving the latter's discretion over the direction of the negotiation process. Negotiations must also be reasonably transparent in terms of outcomes and participation so that the negotiation range can be reliably determined. Breakthroughs taking place in secret or through back-channel negotiations are more difficult to recognize as information regarding these talks is rarely made available publicly or it is being disclosed many years after the fact. Finally, while NBA is good at identifying breakthroughs, it nevertheless fails to explain the scope conditions under which negotiation breakthroughs are more likely to happen. In view of its strong potential to move forward the NBA research agenda, the question of the scope conditions of negotiation breakthroughs ought to be given appropriate scholarly attention in future research. 


\section{CONCLUSIONS}

The aim of this article is to bridge an important gap in the theory of climate negotiations in particular, and of international negotiations more generally namely, the question of negotiation breakthroughs: how to define them conceptually and how to measure them empirically? Drawing on insights offered by market trading theory, I develop a framework of negotiation breakthrough analysis that explains the formation of breakthroughs through the prism of the concept of the negotiation range. More specifically, the negotiation range explains how the outcome of each round of negotiation is being path-dependently shaped by the results of previous meetings and why climate talks have evolved within the rather strict confines of well-defined support and resistance levels. The empirical analysis of the UNFCCC negotiations has found strong support for the technical analytical approach. The PF index has proved itself a valuable instrument for measuring progress in climate talks. It has also reliably identified breaches in the levels of collective expectations of the parties regarding the outcome of climate negotiations. It is also important to note that NBA allows researchers to engage in empirical examinations of negotiation breakthroughs in a highly parsimonious manner. The negotiation range is capable, for instance, of ascertaining the maximal/minimal points of resistance in climate negotiations without taking into account the specific individual preferences or negotiation positions of the parties.

The technical analytical approach has not only theoretical relevance for the study of international negotiations, but it also presents important practical implications. A diplomat attending a multilateral negotiation could use this method for improving his bargaining position in at least two ways. First, NBA can help the diplomat realize that a particular negotiation outcome is no longer business as usual but rather a significant departure from the negotiation pattern. He can translate this insight into a negotiation advantage by choosing when to lead and when to follow others in implementing the results of the talks. Second, he can use the support and resistance levels of the negotiation range to identify 
the issues that are most/less likely to advance the negotiations. For example, by knowing the overall intensity with which a specific policy he favors is being opposed by others, he could adapt his strategy and form coalitions in support of less controversial issues. In so doing, he would be able to build the necessary network of support that could eventually help overturn resistance to the controversial policy in the long-term.

While being instrumental in producing a reliable conceptual framework for recognizing breakthroughs, technical analysis can make, however, a broader contribution to the study of international negotiations. As pointed out above, the concept of negotiation range offers little insight into the scope conditions under which negotiation breakthroughs are likely to happen. This limitation could be addressed through another concept of market analysis, the momentum indicator, which is being used in market trading for assessing the probability of a particular trend to breach the resistance or support level. A third type of application of technical analysis to international negotiations could involve concepts applied by market analysts to confirm the viability of trade breakouts. Context measuring indicators could thus be conceptually translated for NBA to explain why certain potential breakthroughs might fail to materialize. Last but not least, the vast literature on chart patterns developed by market analysts for capturing trend continuation or reversals of stock prices could prove useful for understanding the conditions for breakthrough in environments in which the negotiation range concept might not apply. In short, market analysis offers a promising reservoir of concepts and methods by which to constructively harness the power of complexity for theorizing the evolution and outcome of serial negotiation processes. 


\section{Bibliography:}

Alasuutari, Pertti, Leonard Bickman, and Julia Brannen. 2008. The SAGE handbook of social research methods. Los Angeles: SAGE.

Barret, Scott. 1998. "On the Theory and Diplomacy of Environmental Treaty-Making." Environmental and Resource Economics no. 11 (3-4):317-333.

Barrett, Scott. 2008. "Climate treaties and the imperative of enforcement." Oxford Review of Economic Policy no. 24 (2):239-258.

Biermann, Frank, Philipp H. Pattberg, and Fariborz Zelli. 2010. Global climate governance beyond 2012 : architecture, agency and adaptation. Cambridge: Cambridge University Press.

Carifio, James, and Rocco J. Perla. 2007. "Ten common misunderstandings, misconceptions, persistent myths and urban legends about likert scales and likert response formats and their antidote." Journal of Social Sciences no. 3 (3):106-116.

Chen, James. 2010. Essentials of technical analysis for financial markets. Hoboken, N.J.: Wiley.

Christoff, Peter, and Robyn EcKersley. 2011. "Comparing State Responses." In Oxford handbook of climate change and society, edited by John S. Dryzek, Richard B. Norgaard and David Schlosberg, 431-448. Oxford: Oxford University Press.

City of London. The Total Tax Contribution of UK Financial Services, December, 2010 [Accessed Jan 7, 2013]. Available from http://217.154.230.218/NR/rdonlyres/68F49A7E-8255-415B99A8-1A8273D568D9/0/TotalTax3_FinalForWeb.pdf.

Coleman, Katharina P. 2013. "Locating norm diplomacy: Venue change in international norm negotiations." European Journal of International Relations no. 19 (1):163-186.

Crump, Larry. 2003. "Multiparty Negotiation and the Management of Complexity." International Negotiation no. 8 (2):189-195.

2011. "Negotiation Process and Negotiation Context." International Negotiation no. 16 (2):197-227.

Crump, Larry, and Daniel Druckman. 2012. "Turning Points in Multilateral Trade Negotiations on Intellectual Property." International Negotiation no. 17 (1):9-35.

Crump, Larry, and A. Ian Glendon. 2003. "Towards a Paradigm of Multiparty Negotiation." International Negotiation no. 8 (2):197-234.

Crump, Larry, and I. William Zartman. 2003. "Multilateral Negotiation and the Management of Complexity" no. $8(1): 1-5$.

Downie, Christian. 2012. "Toward an Understanding of State Behavior in Prolonged International Negotiations." International Negotiation no. 17 (2):295-320.

Druckman, Daniel. 2001. "Turning Points in International Negotiation: A Comparative Analysis." The Journal of Conflict Resolution no. 45 (4):519-544.

- 2005. Doing research : methods of inquiry for conflict analysis. Thousand Oaks, Calif.: Sage Publications.

Dunlap, Riley E., and Aaron M. McCright. 2011. "Organized Climate Change Denial." In Oxford 
handbook of climate change and society, edited by John S. Dryzek, Richard B. Norgaard and David Schlosberg, 144-160. Oxford: Oxford University Press.

Fisher, Roger, William Ury, and Bruce Patton. 2011. Getting to yes: negotiating agreement without giving in. 3rd ed. New York: Penguin.

Freestone, David, and Charlotte Streck. 2005. Legal aspects of implementing the Kyoto Protocol mechanisms : making Kyoto work. Oxford ; New York: Oxford University Press.

Gadermann, Anne M., Martin Guhn, and Bruno D. Zumbo. 2012. "Estimating ordinal reliability for Likert-type and ordinal item response data: A conceptual, empirical, and practical guide." Practical Assessment, Research \& Evaluation no. 17 (3):1-3.

Garfinkel, Alan. 1981. Forms of explanation : rethinking the questions in social theory. New Haven, Conn.: Yale University Press.

General Electric. Stock Information: History 2013 [Accessed Jan 24, 2013]. Available from http://www.ge.com/investor-relations/stock-information-history?qm_page=59410.

Goltz, Jan von der. High Stakes in a Complex Game: A Snapshot of the Climate Change Negotiating Positions of Major Developing Country Emitters. Center for Global Development, August, 2009 [Accessed Feb 5, 2011]. Available from http://www.cgdev.org/files/1422602_file_High_Stakes_FINAL081009.pdf.

Granberg-Rademacker, J. Scott. 2010. "An Algorithm for Converting Ordinal Scale Measurement Data to Interval/Ratio Scale." Educational and Psychological Measurement no. 70 (1):74-90.

Hampson, Fen Osler, and Holly Reid. 2003. "Coalition Diversity and Normative Legitimacy in Human Security Negotiations " International Negotiation no. 8 (1):7 - 42.

Harris, Paul G. 2009. Climate change and foreign policy: case studies from east to west. London ; New York: Routledge.

Harstad, Bård. 2010. "How to Negotiate and Update Climate Agreements." In Post-Kyoto international climate policy: implementing architectures for agreement, edited by Joseph E. Aldy and R. N. Stavins, 273-299. Cambridge ; New York: Cambridge University Press.

Hartwig, Frederick, and Brian E. Dearing. 1979. Exploratory data analysis. Beverly Hills: Sage Publications.

Harwell, Michael R., and Guido G. Gatti. 2001. "Rescaling Ordinal Data to Interval Data in Educational Research." Review of Educational Research no. 71 (1):105-131.

Hattori, T. 1999. "The Road to the Kyoto Conference: An Assessment of the Japanese TwoDimensional Negotiation." International Negotiation no. 4 (2):167-195.

Hodgson, Geoffrey M. 2007. "Meanings of methodological individualism." Journal of Economic Methodology no. 14 (2):211-226.

Hoffmann, Matthew J. 2011. Climate governance at the crossroads : experimenting with a global response after Kyoto. Oxford ; New York: Oxford University Press.

Jamieson, S. 2004. "Likert scales: how to (ab)use them." Medical Education no. 38 (12):1217-1218.

Jupp, Victor. 2006. The Sage dictionary of social research methods. London ; Thousand Oaks, Calif.: SAGE Publications.

Kanie, Norichika. 2003. "Leadership in Multilateral Negotiation and Domestic Policy: The 
Netherlands at the Kyoto Protocol Negotiation." International Negotiation no. 8 (2):339-365.

Keohane, Robert O., and Kal Raustiala. 2010. "Toward a Post-Kyoto climate change architrecture: a political analysis." In Post-Kyoto international climate policy : implementing architectures for agreement edited by Joseph E. Aldy and R. N. Stavins, 372-400. Cambridge: Cambridge University Press.

King, Gary, Robert O. Keohane, and Sidney Verba. 1994. Designing social inquiry: scientific inference in qualitative research. Princeton, N.J.: Princeton University Press.

Lewicki, Roy J., Barbara Gray, and Michael Elliott. 2003. Making sense of intractable environmental conflicts : frames and cases. Washington, DC: Island Press.

Marchionni, Caterina. 2008. "Explanatory Pluralism and Complementarity: From Autonomy to Integration." Philosophy of the Social Sciences no. 38 (3):314-333.

Michaelowa, Katharina, and Axel Michaelowa. 2012. "Negotiating climate change." Climate Policy no. $12(5): 527-533$.

Oberthür, Sebastian, and Olav Schram Stokke. 2011. Managing institutional complexity : regime interplay and global environmental change, Institutional dimensions of global environmental change. Cambridge, Mass.: MIT Press.

Putnam, R. D. 1988. "Diplomacy and Domestic Politics - the Logic of 2-Level Games." International Organization no. 42 (3):427-460.

Sandelowski, Margarete, Corrine I. Voils, and George Knafl. 2009. "On Quantitizing." Journal of Mixed Methods Research no. 3 (3):208-222.

Schwager, Jack D. 1999. Getting started in technical analysis. New York: John Wiley.

Sjöstedt, Gunnar, and Ariel Penetrante. 2013. "Strategic Faciliation of Climate Change Negotiation: An Introduction." In Climate change negotiations : a guide to resolving disputes and facilitating multilateral cooperation, edited by Gunnar Sjöstedt, 3-40.

Spector, Bertram. 2003. "Deconstructing the Negotiations of Regime Dynamics." In Getting it Done: Postagreement Negotiation and International Regimes, edited by Bertram Spector and Zartman I. William, 54-87. Washington, D.C.: United States Institute of Peace Press.

Spector, Paul E. 1992. Summated rating scale construction : an introduction, Sage university papers series Quantitative applications in the social sciences. Newbury Park, Calif.: Sage Publications.

Steel, Daniel. 2006. "Methodological Individualism, Explanation, and Invariance." Philosophy of the Social Sciences no. 36 (4):440-463.

Stern, Todd. A New Paradigm: Climate Change Negotiations in the Post-Copenhagen Era, Oct 8, 2010 [Accessed Feb 4, 2011]. Available from

http://www.state.gov/g/oes/rls/remarks/2010/149429.htm.

Susskind, Lawrence E., Boyd W. Fuller, Michèle Ferenz, and David Fairman. 2003. "Multistakeholder Dialogue at the Global Scale." International Negotiation no. 8 (2):235266.

TD Waterhouse. Markets \& Research 2013 [Accessed Jan 24, 2013]. Available from https://www.tdwaterhouse.ca/markets-research/stocks/stocks.jsp.

Udehn, Lars. 2001. Methodological individualism : background, history, and meaning. London ; 
New York: Routledge.

UNFCCC. COP 6-2 Decisions, July, 2001 [Accessed Feb 12, 2013]. Available from http://unfccc.int/meetings/bonn_jul_2001/session/6275/php/view/decisions.php.

. Copenhagen Accord, Dec 18, 2009 [Accessed Jan 10, 2013]. Available from http://unfccc.int/resource/docs/2009/cop15/eng/11a01.pdf\#page=4.

Doha amendment to the Kyoto Protocol, Dec 21, 2012 [Accessed Jan 10, 2013]. Available from http://unfccc.int/files/kyoto_protocol/application/pdf/kp_doha_amendment_english.pdf.

- A Brief Overview of Decisions 2013 [Accessed Feb 12, 2013]. Available from http://unfccc.int/documentation/decisions/items/2964.php.

Vogt, W. Paul. 2005. Dictionary of statistics \& methodology : a nontechnical guide for the social sciences. 3rd ed. Thousand Oaks, Calif.: Sage Publications.

Watkins, Michael. 2003. "Strategic Simplification: Toward a Theory of Modular Design in Negotiation." International Negotiation no. 8 (1):149-167.

Zartman, I. William. 1994. International multilateral negotiation : approaches to the management of complexity. 1st ed. San Francisco: Jossey-Bass.

_ 2003. "Conclusion: Managing Complexity " International Negotiation no. 8 (1):179 - 186. 